\title{
Prediction of Conversion to Alzheimer's Disease with Longitudinal Measures and Time-To-Event Data
}

\author{
Kan $\mathrm{Li}^{\mathrm{a}}$, Wenyaw Chan ${ }^{\mathrm{a}}$, Rachelle S. Doody ${ }^{\mathrm{b}}$, Joseph Quinn ${ }^{\mathrm{c}}$ and Sheng Luo ${ }^{\mathrm{a}, *, 1}$ \\ the Alzheimer's Disease Neuroimaging Initiative ${ }^{1}$ \\ ${ }^{a}$ Department of Biostatistics, The University of Texas Health Science Center at Houston, Houston, TX, USA \\ ${ }^{\mathrm{b}}$ F. Hoffman-La Roche, Basel, Switzerland \\ ${ }^{\mathrm{c}}$ Department of Neurology, Oregon Health and Science University and Portland VA Medical Center, \\ Portland, OR, USA
}

Handling Associate Editor: Jeannie-Marie Leoutsakos

Accepted 5 March 2017

\begin{abstract}
.
Background: Identifying predictors of conversion to Alzheimer's disease (AD) is critically important for AD prevention and targeted treatment.

Objective: To compare various clinical and biomarker trajectories for tracking progression and predicting conversion from amnestic mild cognitive impairment to probable AD.

Methods: Participants were from the ADNI-1 study. We assessed the ability of 33 longitudinal biomarkers to predict time to AD conversion, accounting for demographic and genetic factors. We used joint modelling of longitudinal and survival data to examine the association between changes of measures and disease progression. We also employed time-dependent receiver operating characteristic method to assess the discriminating capability of the measures.

Results: 23 of 33 longitudinal clinical and imaging measures are significant predictors of AD conversion beyond demographic and genetic factors. The strong phenotypic and biological predictors are in the cognitive domain (ADAS-Cog; RAVLT), functional domain (FAQ), and neuroimaging domain (middle temporal gyrus and hippocampal volume). The strongest predictor is ADAS-Cog 13 with an increase of one SD in ADAS-Cog 13 increased the risk of AD conversion by 2.92 times. Conclusion: Prediction of AD conversion can be improved by incorporating longitudinal change information, in addition to baseline characteristics. Cognitive measures are consistently significant and generally stronger predictors than imaging measures.
\end{abstract}

Keywords: ADNI, joint modeling, longitudinal and survival data, mild cognitive impairment, prediction

\footnotetext{
${ }^{1}$ Data used in preparation of this article were obtained from the Alzheimer's Disease Neuroimaging Initiative (ADNI) database (http://adni.loni.usc.edu). As such, the investigators within the ADNI contributed to the design and implementation of ADNI and/or provided data but did not participate in analysis or writing of this report. A complete listing of ADNI investigators can be found at: http://adni.loni.usc.edu/wp-content/ uploads/how_to_apply/ADNI_Acknowledgement_List.pdf.

*Correspondence to: Sheng Luo, PhD, Department of Biostatistics, School of Public Health, The University of Texas Health
}

\section{INTRODUCTION}

Mild cognitive impairment (MCI) often represents an intermediate stage between normal cognition and Alzheimer's disease (AD) [1], and individuals with MCI have been an increasingly common target

Science Center at Houston, 1200 Herman Pressler Dr, Rm E815, Houston, TX 77030, USA. Tel.: +1 713500 9554; E-mail: sheng.t.luo@uth.tmc.edu. 
population for evaluating prognosis and early treatment for AD. However, only a portion of MCI patients progress to dementia while some individuals remain stable or even revert to the normal cognitive status [2]. Identifying predictors of conversion to $\mathrm{AD}$ is therefore critically important for $\mathrm{AD}$ prevention and targeted treatment.

Existing research has implicated a number of biomarkers that predict conversion from MCI to $\mathrm{AD}$ or cognitive decline, including neuroimaging biomarkers [3-6], neuropsychological assessments [7-9], and biomedical biomarkers [10, 11]. Most existing studies $[12,13]$ of predicting time-to-AD adopt Cox regression models with baseline measures. Such an approach implicitly assumes that the predictors stay constant over the length of study, which is unlikely to be true in studies over an extensive period of time. Moreover, the majority of such studies $[14,15]$, exploiting longitudinal measures for predicting future biomarkers or clinical score of MCI patients, fail to take into account dependent terminal events, i.e., a biomarker's trajectory is directly informative about the time to event. In this scenario, separate modeling of the survival outcome and the longitudinal processes may overlook the underlying association and lead to biased inference.

The goal of this research is to identify the optimal outcome measures for enriching an MCI treatment study population with subjects who are most likely to progress over time. Current study designs have become reliant on biomarkers at baseline as a strategy for enriching with decliners, but it remains to be seen whether this strategy will be effective. Incorporation of longitudinal data for subject selection is plausible, and some adaptive design studies have begun to explore this strategy [16-18]. However, the comparative predictive value of longitudinal clinical and imaging data has not been previously reported.

In this paper, instead of considering the conversion of MCI to AD as a binary response, we assessed the ability of various measures to predict time from study entry to AD conversion (first occurrence) for MCI patients using joint modeling of longitudinal and time-to-event data $[19,20]$. The joint model analyzed these two types of outcomes simultaneously and was able to give more accurate parameter estimation and smaller standard errors which in turn yield greater statistical power. The specific measures chosen for comparison in this study covered the domains of clinical measures, neuropsychological assessments, neuroimaging, and functional and behavioral assessments. Relevant demographic and genetic variables (i.e., age, gender, education, and $A P O E$ genotype) were employed as covariates given their potential effects on disease progression in $\mathrm{AD}[9]$.

\section{MATERIALS AND METHODS}

\section{Study design and participants}

Data used in this analysis were obtained from the Alzheimer's Disease Neuroimaging Initiative 1 (ADNI-1) study (http://adni.loni.ucla.edu), which investigates the progression of AD using serial magnetic resonance imaging (MRI), positron emission tomography (PET), other biological markers, and various clinical and neurocognitive measures. Detailed information regarding the ADNI study procedures, including participant inclusion and exclusion criteria and complete study protocol can be found at http://www.adni-info.org. The data are publically available at http://ida.loni.ucla.edu and were downloaded on December 1, 2015.

This analysis included 384 patients with amnestic MCI at baseline evaluation who had at least one follow-up visit. Criteria for MCI were the same as defined by Petersen et al. [1]: a memory complaint that had objective memory loss measured by education adjusted scores on Wechsler Memory Scale Logical Memory II, a Folstein Mini-Mental State Examination score (MMSE) of 24-30, Clinical Dementia Rating (CDR) equal to 0.5, absence of significant levels of impairment in other cognitive domains, and essentially preserved activities of daily living. All subjects were given a written informed consent at the time of enrollment, and the study has been approved by the local institutional review board at all participating sites.

\section{Measures}

The ADNI study collected a broad range of clinical and biological data about patients. We first reviewed the literature and identified 33 measures, based on their availability in the ADNI dataset and sensitivity to detect conversion from $\mathrm{MCI}$ to $\mathrm{AD}$ in prior studies. As part of the ADNI-1, subjects were assessed at baseline, 6, 12, 18, 24, and 36 months, and continued follow-ups were conducted annually as part of the ADNI-2. All potential predictor measures were collected at multiple time points during the follow-up period. We did not consider the domains of cerebrospinal fluid (CSF) biomarkers, because it was 
collected in a small subset of ADNI-1 subjects at baseline and 12 months only.

\section{Neuropsychological assessment}

Measurements in the neuropsychological domain included the Alzheimer's Disease Assessment Scale-Cognitive (ADAS-Cog), which assesses written and verbal responses of subjects that are related to fundamental cognitive functions. The total score is reported as a composite score of 11 or expanded to 13 items and ranges from 0 to 70 or 85 , with a higher score indicating poor cognitive function. Other measures of verbal memory included were the Rey Auditory Verbal Learning Test (RAVLT immediate, RAVLT learning, RAVLT forgetting), the MMSE (11 questions with scores range from 0 to 30 and lower scores reflect more severe cognitive impairment), Montreal Cognitive Assessment (MoCA; a 30-point test assesses different cognitive domains), and CDR Sum of Boxes (CDR-SB; sum of box method to stage severity of dementia with range from 0 to 18 ).

\section{Functional and behavioral assessment}

Variables included Functional Assessment Questionnaire (FAQ; 10 items with scores range from 0 to 30 , with higher scores reflecting greater functional dependence), Everyday Cognition by the patient (ECogPt), and Everyday Cognition by the patient's study partner (ECogSP), which assesses participant's capability of performing normal everyday tasks in multiple domains (Memory, Language, Visuospatial Abilities, Planning, Organization, Divided Attention, and Total score).

\section{Neuroimaging}

Neuroimaging measures were PET imaging results including FDG-PET, PIB, and AV45, and MRI volumetric data of ventricles, hippocampus, whole brain, entorhinal, fusiform gyrus, middle temporal gyrus, and intracerebral volume. The analyzed data from previous studies [21-24] were used. FDG-PET was represented as a sum of mean glucose metabolism uptake in regions of angular (right and left), temporal (right and left), and posterior cingulate [21]. PIB was the average of standardized uptake value (SUVR) of frontal cortex, anterior cingulate, precuneus cortex, and parietal cortex [22]. AV45 was the average of florbetapir SUVR of frontal, anterior and posterior cingulate, lateral parietal, and lateral temporal cortex [23]. The detailed protocol of ADNI PET data acquisition and processing are available at http://adni.loni.usc.edu/data-samples/pet/. The MRI data were acquired on $1.5 \mathrm{~T}$ or $3 \mathrm{~T}$ MRI scanners, and volumes of the region of interests (ROIs) were reconstructed with the Freesurfer [24].

\section{Statistical analyses}

The markers in this analysis were collected from participants at multiple time points during the followup period and were hypothesized to be related to $\mathrm{AD}$ progression - the event of interest. When the value of a marker at a time point is affected by the occurrence of an event at that time point, the longitudinal marker is an endogenous time-dependent covariate. However, the Cox model and its extensions cannot properly handle endogenous covariates [25]. We simultaneously modeled time-to-dementia as well as longitudinal change in the aforementioned neuropsychological, neuroimaging, or functional/behavioral variables, using joint modelling for longitudinal and survival data. A joint model consisted of two submodels: the longitudinal sub-model and the survival sub-model. The longitudinal sub-model allowed us to describe the evolution of a repeated measure over time, while adjusting for age at baseline and presence of apolipoprotein $\mathrm{E}(A P O E) \varepsilon 4$ allele. A random intercept and a random slope of time were also included in the sub-model to capture the between subject variation. We assumed linear trajectories for the markers, because the longitudinal measures under consideration did not display highly non-linearity in the trajectories before their AD conversion (Figs. 1 and 2). However, in a different setting where patients could have highly non-linear evolution, spline could be implemented in the longitudinal model to account for the nonlinear trajectories of measures. The survival time (in years) was defined as from the baseline visit date to $\mathrm{AD}$ conversion or censoring. Although certain participants were continued followed after the event of interest in the original study, we excluded the visits and measurements after $\mathrm{AD}$ conversion from this analysis. The survival sub-model took the form of a proportional hazards model with baseline covariates such as gender, age at baseline, presence of the $A P O E \varepsilon 4$ allele, years of education, and one of the longitudinal measures. An association parameter $(\alpha)$ linked the two sub-models, assuming the hazard was dependent on the longitudinal measure through its current value.

Suppose $y_{i}\left(t_{i j}\right)$ were the observations of one of the longitudinal measurements for the $i$ th person $(i=1, \ldots, N)$ at the $j$ th time point $\left(j=1, \ldots, n_{i}\right)$, $t_{i j}$. The joint model was represented as 

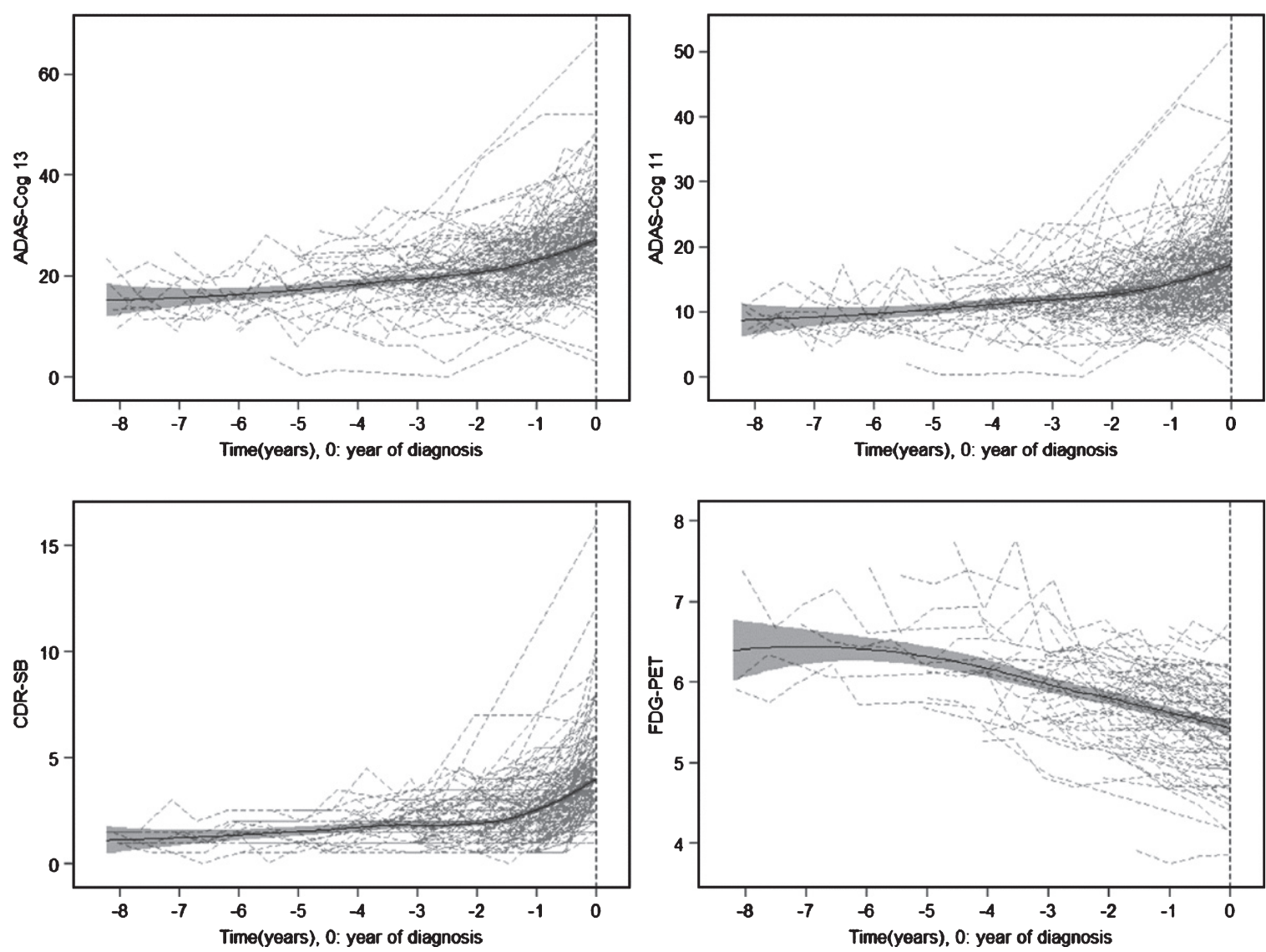

Fig. 1. Trajectories of ADAS-Cog 13, ADAS-Cog 11, CDR-SB, and FDG-PET before AD conversion for the 200 participants who progressed to AD during the study. Dashed lines (light gray) are individual empirical data and solid lines are cubic spline curves (shading shows $95 \%$ CIs). The vertical line in each panel denotes year of AD diagnosis (set to year 0). ADAS-Cog, Alzheimer's Disease Assessment Scale-Cognitive Subscale test; CDR-SB, Clinical Dementia Rating Sum of Boxes; FDG-PET, sum of mean glucose metabolism uptake in regions of angular, temporal, and posterior cingulate.

$$
\begin{aligned}
y_{i}\left(t_{i j}\right)= & m_{i}\left(t_{i j}\right)+\varepsilon_{i}\left(t_{i j}\right), \text { where } \\
m_{i}\left(t_{i j}\right)= & \beta_{0}+\beta_{1} t_{i j}+\beta_{2} \text { baselineAge }_{i} \\
& +\beta_{3} i . A P O E \varepsilon 4_{i}+b_{0 i}+b_{1 i} t_{i j}, \text { and } \\
h_{i}(t)= & h_{0}(t) \exp \left\{\gamma_{1} \text { gender }_{i}+\gamma_{2} \text { beselineAge }_{i}\right. \\
& \left.+\gamma_{3} \text { education }_{i}+\gamma_{4} i . A P O E \varepsilon 4_{i}+\alpha m_{i}(t)\right\} .
\end{aligned}
$$

The parameter $b_{0 i}$ was the random intercept, $b_{1 i}$ was the random slope indicating subject-specific change rate of the measure, and $\varepsilon_{i}\left(t_{i j}\right)$ was the measurement error. A significant $\alpha$ indicated a strong association between the longitudinal measure and time to AD conversion. The joint model was reduced to separate models if $\alpha=0$, and the advantage of joint model disappeared. The quantity $\exp (\alpha)$ was the hazard ratio (HR; inverse hazard ratio $\mathrm{HR}^{-1}$ was used when the $\alpha$ estimate was negative) for a one-unit increase in the trajectory $m_{i}(t)$, at time $t$. Specifically, a one-unit increase in trajectory increased the hazard by $\exp (\alpha)$ times. To facilitate the comparison of the association parameter among markers, each maker was scaled to zero-mean and unit variance using the mean and standard deviation (SD) among all the participants and all the time points. For comparison, we also fitted a cross-sectional proportional hazards model (Cox model) that only incorporated the baseline measure in the prediction. The cross-sectional Cox model was represented as

$$
\begin{aligned}
h_{i}(t)= & h_{0}(t) \exp \left\{\gamma_{1} \text { gender }_{i}+\gamma_{2} \text { baselineAge }_{i}\right. \\
& \left.+\gamma_{3} \text { edication }_{i}+\gamma_{4} \text { i.APOE } 4_{i}+\alpha y_{i 1}\right\},
\end{aligned}
$$

where $h_{0}(t)$ was the baseline hazard function and $y_{i 1}$ was the measure taken at the baseline visit. The parameter $\alpha$ in the Cox model quantified the association between the baseline measurement and the event hazard. We refer the readers to [26] for an 
introductory overview on joint modeling and the comparison with Cox model. For consistency, we scaled each marker at baseline to zero-mean and unit variance.

We also assessed how well these measures could discriminate between MCI patients who progressed to $\mathrm{AD}$ and those who had a stable status. We calculated time-dependent areas under the ROC curves (AUCs) to assess the performance of the longitudinal marker at different time points over the follow-up period. We also computed the dynamic discrimination index (DDI) [27], which summarized the discrimination power of the measure over the whole follow-up period for a $\Delta t$ ahead prediction, using a weighted average of AUCs. In general, higher AUCs and DDI indicate higher discrimination of the models. We computed the probabilities of conversion to probable AD in the time frame $(t, t+\Delta t]$, which meant using all measures of subject survived till time $t$ to perform a $\Delta t$ ahead prediction. We selected $t$ at 12 , 18 , and 24 months, and $\Delta t$ as 6 and 12 months.

To avoid overestimation of the predictive performance of the markers [28], we conducted a $k$-fold cross validation. The total sample of the MCI patients was randomly broken into $k$ subgroups of approximately equal sizes. The analysis was repeated $k$ times with one subset being left out as the test set and the others being used as the training set in each analysis. Parameter estimates of the joint model were derived from the training set and applied to the test set. Predictive accuracies were then computed by averaging the $k$ separate analyses. We used $k$ as 6 to have about 320 subjects in the training set and 64 subjects in the test set, which was considered necessary for power and allowed for a reasonable number of validations.

All the analyses were performed using R (version $3 \cdot 2 \cdot 1)$. Cross-sectional Cox modeling and proportionality testing were conducted using the survival package. The Joint modeling and time-dependent AUCs calculation were achieved using the JM package [29].

\section{RESULTS}

The demographic characteristics of the study population are shown in Table 1. Participants were followed up for a mean of 3.2 years (SD 2.6; range 0.4-9.3 years) before conversion to $\mathrm{AD}$ or censoring. The average age at baseline was $74.7,37.5 \%$ were women and had a 15.6-year average education length. More than $54.2 \%$ of subjects had one or more APOE $\varepsilon 4$ alleles. Among the $384 \mathrm{MCI}$ patients, 200 patients converted to AD over a mean follow-up period of 2.3 years. 184 patients had stable MCI, of which 77 had less than 3 years of follow-up while 107 were followed for at least 3 years. $23 \%$ subjects had only one follow-up visit.

Table 2 shows the results of two models for each measure. We conducted the $\mathrm{Z}$ test of the null hypothesis in which the association parameter was zero (no association between maker and time to AD conversion). The HR was the primary effect size, and the markers were ranked based on the absolute $\mathrm{Z}$ value of the association parameter $\alpha$ from the longitudinal prediction model. The column of the association parameter for the cross-sectional Cox model indicates that the baseline information was a significant predictor of the hazard of AD progression for 19 of the 33 measures. The majority of the association parameters in the longitudinal model were larger (in absolute value) than their counterparts in the cross-sectional Cox model, where 23 out of 33 measures were significantly predictors of AD conversion. The longitudinal measures of ECogSP (in the domains of language, planning and visuospatial abilities) and RAVLT forgetting were significant predictors, although their baseline measures were not significant in the cross-sectional Cox model. Thus, prediction of $\mathrm{AD}$ progression based on both baseline and longitudinal changes was stronger than prediction based only on baseline information. Based on the results of the joint models, the strongest predictors were ADAS-Cog 13 and ADAS-Cog 11, followed by RAVLT immediate, FAQ, and middle temporal gyrus volume. Specifically, an increase of one SD (7.7 units) in trajectory of ADAS-Cog 13 score increases the hazard of $\mathrm{AD}$ conversion by 2.92 times $(95 \% \mathrm{CI}$ 2.33-3.66), a decrease of one SD (10.6 units) in trajectory of RAVLT immediate recall increases hazard by 3.16 times (95\% CI 2.41-4.15), an increase of one SD (5.2 units) in trajectory of FAQ score increases hazard of $\mathrm{AD}$ conversion by 1.95 times $(95 \% \mathrm{CI}$ 1.64-2.34), and a reduction of one $\operatorname{SD}\left(2851 \mathrm{~mm}^{3}\right)$ in middle trajectory of temporal gyrus volume increases hazard by 1.98 times (95\% CI 1.65-2.37).

Figure 1 shows individual empirical curves and fitted spline curves before AD conversion for the participants who progressed to $\mathrm{AD}$ during the study, for ADAS-Cog 13, ADAS-Cog 11, CDR-SB, and FDG-PET, respectively. Dashed lines are individual empirical data and solid lines are cubic spline curves (shading shows 95\% CIs). The vertical line in each panel denotes the year of AD diagnosis (set to year 0 ). 
Table 1

Baseline characteristics of ADNI-1 participants with mild cognitive impairment

\begin{tabular}{lccc}
\hline & $\begin{array}{c}\text { Progressed to AD } \\
\text { during the study } \\
(n=200)\end{array}$ & $\begin{array}{c}\text { Did not progress to } \\
\text { AD during the study } \\
(n=184)\end{array}$ & $\begin{array}{c}\text { Combined } \\
(n=384)\end{array}$ \\
\hline Women & $75(37.50 \%)^{*}$ & $62(33.50 \%)$ & $137(35.7 \%)$ \\
Age (years) & $74.44(7.09)$ & $75.03(7.55)$ & $74.71(7.31)$ \\
APOE4 present & $127(63.50 \%)$ & $81(44.00 \%)$ & $208(54.16 \%)$ \\
Education (years) & $15.82(2.86)$ & $15.33(3.19)$ & $15.58(3.03)$ \\
Time in study (years) & $2.25(1.74)$ & $4.24(2.91)$ & $3.20(2.57)$ \\
\hline
\end{tabular}

*Data are mean (SD) or $n(\%)$.

Table 2

Prediction of risk of AD progression: model results (controlled for age, APOE in the longitudinal model and controlled for age, gender, years of education and $A P O E$ in the survival model)

\begin{tabular}{|c|c|c|c|c|c|c|c|c|c|}
\hline \multirow[b]{2}{*}{ Variable* } & \multirow[b]{2}{*}{ Participants } & \multirow[b]{2}{*}{ Observations } & \multirow[b]{2}{*}{ Events ${ }^{\dagger}$} & \multicolumn{3}{|c|}{$\begin{array}{l}\text { Cross-sectional prediction } \\
\text { (Cox Model) }\end{array}$} & \multicolumn{3}{|c|}{$\begin{array}{l}\text { Longitudinal prediction } \\
\text { (Joint Model) }\end{array}$} \\
\hline & & & & $\alpha$ & $\begin{array}{c}\mathrm{Z} \\
\text { value }\end{array}$ & $\begin{array}{c}\mathrm{HR}^{\ddagger} \text { or } \mathrm{HR}^{-1} \\
(95 \% \mathrm{CI})\end{array}$ & $\alpha$ & $\begin{array}{r}Z \\
\text { value }\end{array}$ & $\begin{array}{c}\mathrm{HR}^{\ddagger} \text { or } \mathrm{HR}^{-1} \\
(95 \% \mathrm{CI})\end{array}$ \\
\hline ADAS-Cog 13 & 384 & 1872 & 200 & 0.95 & 9.08 & $2.57(2.10-3.16)$ & 1.07 & 9.33 & $2.92(2.33-3.66)$ \\
\hline ADAS-Cog 11 & 384 & 1890 & 200 & 0.81 & 8.18 & $2.25(1.85-2.74)$ & 0.92 & 8.40 & $2.52(2.03-3.12)$ \\
\hline RAVLT.immediate & 384 & 1883 & 200 & -0.85 & -8.26 & $2.34(1.92-2.85)$ & -1.15 & -8.32 & $3.16(2.41-4.15)$ \\
\hline FAQ & 384 & 1895 & 200 & 0.59 & 8.06 & $1.80(1.56-2.07)$ & 0.67 & 7.38 & $1.95(1.64-2.34)$ \\
\hline MidTemp & 362 & 1290 & 184 & -0.64 & -7.22 & $1.90(1.59-2.26)$ & -0.68 & -7.31 & $1.96(1.65-2.37)$ \\
\hline RAVLT.learning & 384 & 1883 & 200 & -0.37 & -4.61 & $1.44(1.23-1.68)$ & -1.24 & -6.45 & $3.47(2.38-5.06)$ \\
\hline MMSE & 384 & 1888 & 200 & -0.57 & -4.78 & $1.76(1.40-2.23)$ & -0.58 & -6.43 & $1.79(1.50-2.13)$ \\
\hline Hippocampus & 362 & 1296 & 184 & -0.60 & -6.49 & $1.83(1.52-2.20)$ & -0.60 & -6.30 & $1.82(1.51-2.19)$ \\
\hline CDR-SB & 384 & 1892 & 200 & 0.75 & 6.08 & $2.11(1.66-2.69)$ & 0.66 & 6.30 & $1.93(1.57-2.37)$ \\
\hline FDG-PET & 220 & 905 & 100 & -0.56 & -4.65 & $1.76(1.38-2.22)$ & -0.74 & -6.08 & $2.11(1.66-2.68)$ \\
\hline Entorhinal & 362 & 1290 & 184 & -0.49 & -5.62 & $1.64(1.38-1.95)$ & -0.61 & -6.00 & $1.84(1.51-2.25)$ \\
\hline Fusiform & 362 & 1290 & 184 & -0.43 & -4.83 & $1.53(1.29-2.26)$ & -0.50 & -5.33 & $1.64(1.37-1.98)$ \\
\hline EcogSPTotal & 79 & 282 & 13 & 1.01 & 2.85 & $2.75(1.37-5.51)$ & 1.34 & 3.24 & $3.83(1.70-8.63)$ \\
\hline WholeBrain & 383 & 1612 & 199 & -0.33 & -3.46 & $1.40(1.16-1.69)$ & -0.32 & -3.21 & $1.38(1.13-1.68)$ \\
\hline EcogSPMem & 80 & 283 & 13 & 0.99 & 3.02 & $2.70(1.42-5.14)$ & 1.37 & 3.14 & $3.93(1.67-9.22)$ \\
\hline EcogSPLang & 80 & 285 & 13 & 0.55 & 1.82 & $1.73(0.96-3.11)$ & 1.11 & 2.96 & $3.03(1.46-6.32)$ \\
\hline EcogSPDivatt & 79 & 264 & 13 & 0.97 & 2.59 & $2.63(1.27-5.49)$ & 1.54 & 2.93 & $4.67(1.67-13.09)$ \\
\hline EcogSPOrgan & 78 & 273 & 13 & 0.72 & 2.29 & $2.06(1.11-3.81)$ & 0.98 & 2.85 & $2.66(1.36-5.22)$ \\
\hline EcogSPPlan & 79 & 277 & 13 & 0.49 & 1.45 & $1.64(0.84-3.20)$ & 1.20 & 2.66 & $3.33(1.37-8.08)$ \\
\hline RAVLT.forgetting & 384 & 1878 & 200 & 0.12 & 1.62 & $1.13(0.97-1.30)$ & 0.45 & 2.65 & $1.57(1.13-2.20)$ \\
\hline EcogSPVisspat & 77 & 274 & 12 & 0.64 & 1.92 & $1.90(0.99-3.66)$ & 0.93 & 2.49 & $2.53(1.22-5.25)$ \\
\hline Ventricles & 383 & 1597 & 199 & 0.20 & 2.57 & $1.22(1.05-1.42)$ & 0.18 & 2.37 & $1.19(1.03-1.38)$ \\
\hline AV45-PET & 58 & 104 & 10 & 0.74 & 2.00 & $2.09(1.02-4.31)$ & 0.76 & 2.01 & $2.14(1.02-4.48)$ \\
\hline EcogPtOrgan & 79 & 285 & 13 & 0.44 & 1.81 & $1.56(0.96-2.52)$ & 0.65 & 1.95 & $1.91(1.00-3.68)$ \\
\hline EcogPtMem & 80 & 290 & 13 & 0.48 & 1.81 & $1.61(0.96-2.70)$ & 0.71 & 1.92 & $2.03(0.99-4.16)$ \\
\hline EcogPtTotal & 80 & 290 & 13 & 0.34 & 1.35 & $1.41(0.86-2.33)$ & 0.52 & 1.66 & $1.69(0.91-3.13)$ \\
\hline EcogPtDivatt & 80 & 289 & 13 & 0.21 & 0.80 & $1.23(0.74-2.04)$ & 0.47 & 1.36 & $1.61(0.81-3.17)$ \\
\hline MOCA & 77 & 274 & 13 & -0.22 & -0.64 & $1.25(0.63-2.46)$ & -0.50 & -1.34 & $1.66(0.79-3.46)$ \\
\hline PIB-PET & 60 & 113 & 27 & 0.24 & 1.05 & $1.27(0.81-1.99)$ & 0.32 & 1.31 & $1.37(0.85-2.21)$ \\
\hline EcogPtLang & 80 & 289 & 13 & 0.26 & 0.94 & $1.30(0.75-2.25)$ & 0.39 & 1.08 & $1.48(0.73-3.01)$ \\
\hline EcogPtPlan & 80 & 290 & 13 & 0.07 & 0.29 & $1.08(0.65-1.77)$ & 0.42 & 0.99 & $1.52(0.66-3.48)$ \\
\hline EcogPtVisspat & 80 & 286 & 13 & 0.04 & 0.12 & $1.04(0.56-1.93)$ & 0.30 & 0.93 & $1.34(0.72-2.50)$ \\
\hline ICV & 384 & 1632 & 200 & 0.01 & 0.10 & $1.01(0.84-1.21)$ & 0.00 & 0.05 & $1.00(0.83-1.21)$ \\
\hline
\end{tabular}

* Measures are ranked by absolute Z-value for the association parameter $(\alpha)$ of the Joint Model. ${ }^{\dagger}$ Events are the number of conversions to AD. ${ }^{\ddagger}$ Hazard ratio (HR) or inverse hazard ratio $\left(\mathrm{HR}^{-1}\right)$ show the hazard for $\mathrm{AD}$ diagnosis associated with a difference of one $\mathrm{SD}$ in each measure. The variable descriptions are provided in the Supplementary material. ADAS-Cog, Alzheimer's Disease Assessment Scale - Cognitive Subscale test; RAVLT, Rey Auditory Verbal Learning Test; FAQ, Functional Assessment Questionnaire; MidTemp, middle temporal gyrus; MMSE, Mini-Mental State Examination; CDR-SB, Clinical Dementia Rating Sum of Boxes; FDG-PET, sum of mean glucose metabolism uptake in regions of angular, temporal, and posterior cingulate; ECogPt, Everyday Cognition questionnaire filled out by the patient; ECogSP, Everyday Cognition questionnaire filled out by the patient's study partner; MOCA, Montreal Cognitive Assessment; ICV, intracerebral volume. 

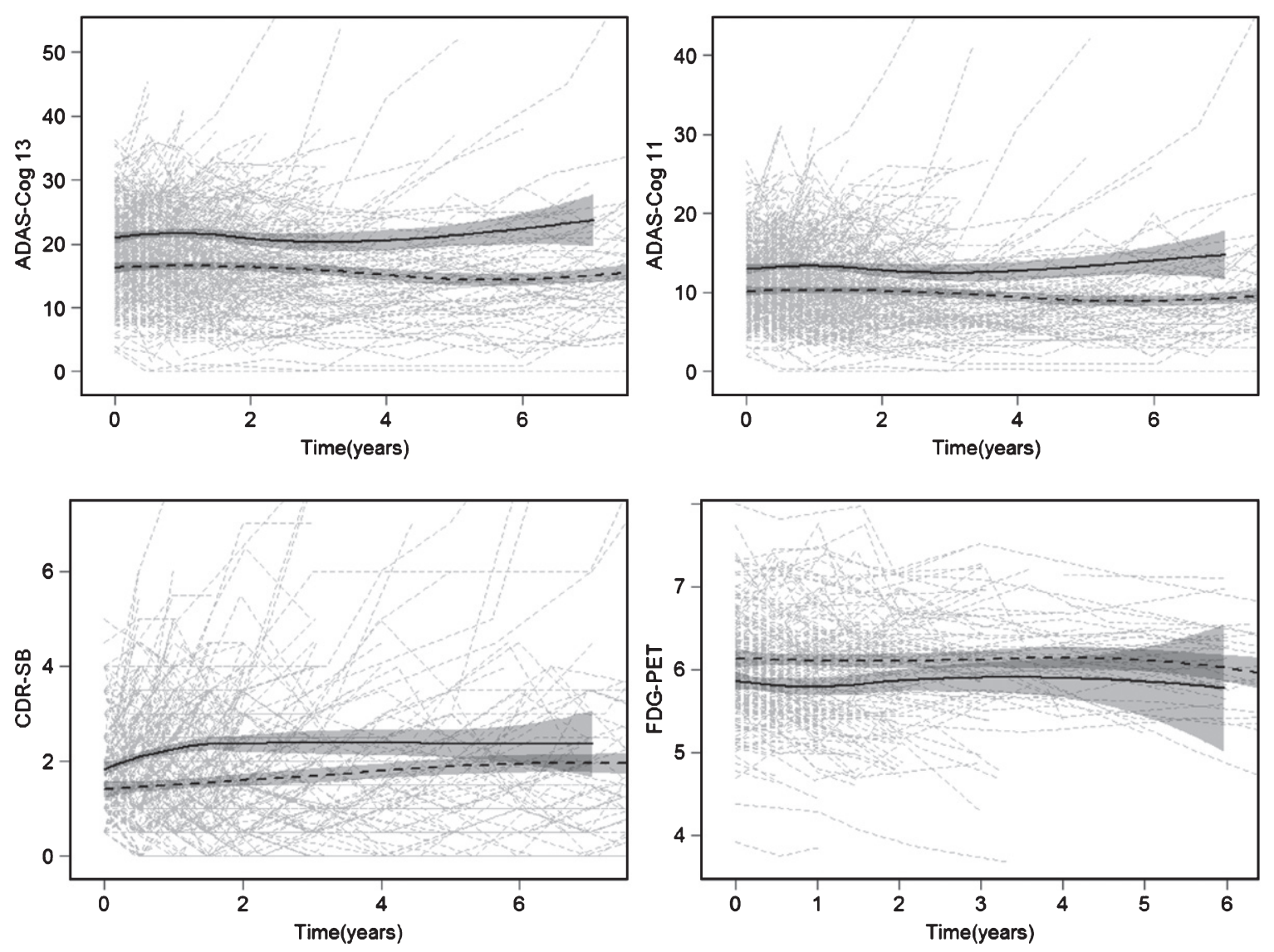

Fig. 2. Trajectories of ADAS-Cog 13, ADAS-Cog 11, CDR-SB, and FDG-PET for all participants. Dashed lines (light gray) are individual empirical data. The solid lines represent participants converted to AD during the study and the wide dashed lines represent the participants not converted to AD during the study (shading shows 95\% CIs). The two groups can be clearly distinguished by each of these measures. 0 denotes year of entry the study. ADAS-Cog, Alzheimer's Disease Assessment Scale-Cognitive Subscale test; CDR-SB, Clinical Dementia Rating Sum of Boxes; FDG-PET, sum of mean glucose metabolism uptake in regions of angular, temporal, and posterior cingulate.

The figure shows that all measures deteriorated as AD progressed. Figure 2 shows the trajectories and fitted spline curves for participants who progressed and those who did not progress to AD, for ADAS-Cog 13, ADAS-Cog 11, CDR-SB, and FDG-PET, respectively. Only measurements before AD conversion or censoring are plotted on the figure. Participants who progressed to $\mathrm{AD}$ and those who did not progressed to $\mathrm{AD}$ during the study can be clearly distinguished by each of these measures.

Table 3 compares the discriminative capability of the top 10 strongest predictors by calculating the time-dependent AUCs at the 12th, 18th, and 24th month. Different from just using baseline predictors, the prediction in the joint model was based on accumulating evidence. Specifically, the values in the first column in Table 3 evaluated the performance of using all previous observations of the remaining MCI patients (those who had not progress to AD) at 12 months (sample size $n=315$ ) to predict their disease status between the 12th and 18th month. The DDI summarizes the discrimination power of the marker to predict the patients' disease status in the next 6 or 12 months. Among the predictors, ADAS-Cog 13 had the best discrimination performance with AUCs ranging from 0.74 to 0.86 for all combinations of $t$ and $\Delta t$, and DDI being 0.79 and 0.79 for $\Delta t$ as 6 and 12 months, respectively. In general, cognitive and functional markers (ADAS, Auditory Verbal Learning Test, FAQ, and MMSE) have higher AUCs and DDI than those of imaging markers, indicating that cognitive measures may be more useful in predicting risk of $\mathrm{AD}$ conversion within a few years among MCI patients. 
Table 3

Areas under the ROC curve and estimated dynamic discrimination index*

\begin{tabular}{|c|c|c|c|c|c|c|c|}
\hline \multirow{2}{*}{$\begin{array}{l}\Delta t^{\ddagger} \text { (month) } \\
t^{\dagger} \text { (month) } \\
\text { (n) }\end{array}$} & & \multicolumn{3}{|c|}{6} & \multicolumn{3}{|c|}{12} \\
\hline & & $\begin{array}{c}12 \\
(n=315)\end{array}$ & $\begin{array}{c}18 \\
(n=265)\end{array}$ & $\begin{array}{c}24 \\
(n=217)\end{array}$ & $\begin{array}{c}12 \\
(n=315)\end{array}$ & $\begin{array}{c}18 \\
(n=265)\end{array}$ & $\begin{array}{c}24 \\
(n=217)\end{array}$ \\
\hline \multirow[t]{2}{*}{ ADAS-Cog 13} & AUC & 0.80 & 0.74 & 0.86 & 0.78 & 0.78 & 0.81 \\
\hline & DDI & & 0.79 & & & 0.79 & \\
\hline \multirow[t]{2}{*}{ ADAS-Cog 11} & AUC & 0.79 & 0.72 & 0.86 & 0.76 & 0.77 & 0.79 \\
\hline & DDI & & 0.78 & & & 0.78 & \\
\hline \multirow[t]{2}{*}{ RAVLT. Immediate } & AUC & 0.78 & 0.76 & 0.85 & 0.78 & 0.79 & 0.83 \\
\hline & DDI & & 0.78 & & & 0.76 & \\
\hline \multirow[t]{2}{*}{ FAQ } & AUC & 0.79 & 0.74 & 0.81 & 0.78 & 0.75 & 0.77 \\
\hline & DDI & & 0.74 & & & 0.72 & \\
\hline \multirow[t]{2}{*}{ MidTemp } & AUC & 0.71 & 0.73 & 0.71 & 0.74 & 0.72 & 0.68 \\
\hline & DDI & & 0.67 & & & 0.69 & \\
\hline \multirow[t]{2}{*}{ RAVLT. Learning } & AUC & 0.74 & 0.69 & 0.78 & 0.72 & 0.71 & 0.73 \\
\hline & DDI & & 0.74 & & & 0.71 & \\
\hline \multirow[t]{2}{*}{ MMSE } & AUC & 0.74 & 0.72 & 0.83 & 0.74 & 0.74 & 0.75 \\
\hline & DDI & & 0.75 & & & 0.72 & \\
\hline \multirow[t]{2}{*}{ Hippocampus } & AUC & 0.73 & 0.66 & 0.74 & 0.70 & 0.71 & 0.69 \\
\hline & DDI & & 0.68 & & & 0.69 & \\
\hline \multirow[t]{2}{*}{ CDR-SB } & AUC & 0.77 & 0.73 & 0.80 & 0.77 & 0.74 & 0.74 \\
\hline & DDI & & 0.73 & & & 0.72 & \\
\hline \multirow[t]{2}{*}{ FDG-PET } & AUC & 0.76 & 0.68 & 0.77 & 0.72 & 0.70 & 0.68 \\
\hline & DDI & & 0.70 & & & 0.71 & \\
\hline
\end{tabular}

*The top 10 strongest predictors. AUC is the area under the ROC curve and DDI is dynamic discrimination index. Higher value indicates higher discrimination capability. ${ }^{\dagger} t$ is follow-up time, corresponding to different amounts of available longitudinal information. $n$ is the remaining MCI patients at time $t$. Predictive utility is evaluated based on the longitudinal measure of these $n$ patients observed before time $t .{ }^{\ddagger} \Delta t$ is relevant time window, corresponding to different prediction intervals. ADAS-Cog, Alzheimer's Disease Assessment Scale - Cognitive Subscale test; RAVLT, Rey Auditory Verbal Learning Test; FAQ, Functional Assessment Questionnaire; MidTemp, middle temporal gyrus; MMSE, Mini-Mental State Examination; CDR-SB, Clinical Dementia Rating Sum of Boxes; FDG-PET, sum of mean glucose metabolism uptake in regions of angular, temporal, and posterior cingulate.

\section{DISCUSSION}

In this paper, we used comprehensive longitudinal assessments to predict dementia in AD in a manner that has not been accomplished by prior studies. By accounting for dependent terminal events, the joint models of longitudinal change and time to AD conversion identified several significant predictors. The strongest predictors are in the cognitive domain (ADAS-Cog, RAVLT), functional domain (FAQ), and neuroimaging domain (middle temporal gyrus and hippocampal volume). These findings are consistent with reports in the literature [3, 4, 6-9].

Our study has also consolidated the findings in Fleisher et al. [30] in the sense that common cognitive measures could provide more accurate prediction regarding AD conversion than volumetric MRI measures by evaluating the discriminative capability of the measures at different time points. In a large metaanalysis which did not include ADNI data, baseline cognitive measures were demonstrated as better predictors of $\mathrm{AD}$ conversion than brain volumetric markers [31]. Thus, with consideration of the comparative economy of cognitive measures, in expense and time, these measures should still be the gold standard for clinical assessment of conversion from MCI to AD. In addition, we note that different markers may show different predictive values at different times in disease progression. This has been reflected in the different changes of AUCs with the passage of time. The cognitive measures such as ADAS-Cog retain a moderate discriminative capability even in the later phrase of disease process while neuroimaging measures (volumetric MRI, PET) become less useful as time passes. This may explain why volumetric changes on MRI were reported to be better predictors than cognitive measures among cognitively normal individuals [32]. A similar point was also made in the literature [33, 34]. Our analyses controlled for the presence of $A P O E$ and demographic variables that have been associated with cognitive decline or the likelihood of developing AD. One caveat in our study is that some neuropsychological tests and clinical measures, such as CDR-SB, may 
be used in the diagnostic process of AD. However, diagnoses are based, in various weight, on clinical history, laboratory data, and a full battery of tests which include some of the selected measures. It is also known that how far someone has progressed on a staging or cognitive measure predicts how fast they decline. Therefore, it is reasonable to explore selected measures as predictors in the joint models.

There are several limitations in our study. First, not all ADNI-1 participants underwent all measurements examinations, especially PET imaging and Everyday Cognition. The differences in sample sizes, particularly the smaller sample of FDG-PET and PIB-PET, may limit our ability to compare their predictive capabilities. However, this did not affect our conclusion on the other markers. Second, while each measure independently showed promise in predicting disease progression in our study, many recent researchers have shown interest in examining biomarker combinations as predictors for AD conversion [35-37]. The joint model adopted in present analysis can only handle a single longitudinal outcome, but can be extended to incorporate multivariate longitudinal measures as proposed in $\mathrm{He}$ et al. [38]. The general idea is to introduce a continuous latent variable to represent patients' underlying disease severity. The observed longitudinal markers can be modeled as measurements of the latent variable using a multilevel item response theory sub-model and the time-to-event data are modeled using a Cox proportional hazard sub-model. Because all outcomes share the same latent variable, the dimensionality of the data can be reduced and fewer parameters are needed. Wang et al. [39] proposed a prediction framework for multiple longitudinal measures and event time data based on the method. Simultaneous modeling of multiple longitudinal outcomes in joint models may substantially enhance the predictive ability of a joint model, and help to identifying the optimal combination of measures in determining the risk of incident $\mathrm{AD}$ dementia in MCI patients. Moreover, rather than using the diagnoses assigned by ADNI, which has been shown to produce a high rate of false positive diagnostic errors, the new approach for staging preclinical AD [40] would sharpen our model to identify early predictors. Last, the ADNI cohort is a convenience sample rather than an epidemiologic cohort; it is likely to result in recruiting more impaired subjects. However, this study focused on MCI population and may be less prone to selection bias than the larger ADNI study population. The cross-validation used in the analysis was an internal validation, and external validation with an independent data set could further consolidate our findings.

In summary, our study was the first attempt to comprehensively and systematically evaluate the predictive ability of markers for $\mathrm{AD}$ conversion under the joint model framework, which includes both baseline measures and changes in these measures over time. The sample from ADNI that we used was large, and the data were collected uniformly, rigorously, and on a broad range of measures. We demonstrated that cognitive measures were consistently significant and generally stronger predictors than imaging measures, with ADAS-Cog 13 as the optimal predictor. Moreover, the measures identified as strong predictors in this study along with each joint model can be used for subject specific prediction. Such individualized risk prediction can help personalize screening strategies and/or guide the initiation of treatment among MCI patients or subject selection for clinical trials.

\section{ACKNOWLEDGMENTS}

This work was supported by the National Institute of Neurological Disorders and Stroke (R01NS 091307 and 5U01NS043127).

Data collection and sharing for this project was funded by the Alzheimer's Disease Neuroimaging Initiative (ADNI) (National Institutes of Health Grant U01 AG024904) and DOD ADNI (Department of Defense award number W81XWH-12-2-0012). ADNI is funded by the National Institute on Aging, the National Institute of Biomedical Imaging and Bioengineering, and through generous contributions from the following: AbbVie, Alzheimer's Association; Alzheimer's Drug Discovery Foundation; Araclon Biotech; BioClinica, Inc.; Biogen; BristolMyers Squibb Company; CereSpir, Inc.; Cogstate; Eisai Inc.; Elan Pharmaceuticals, Inc.; Eli Lilly and Company; EuroImmun; F. Hoffmann-La Roche Ltd and its affiliated company Genentech, Inc.; Fujirebio; GE Healthcare; IXICO Ltd.; Janssen Alzheimer Immunotherapy Research \& Development, LLC.; Johnson \& Johnson Pharmaceutical Research \& Development LLC.; Lumosity; Lundbeck; Merck \& Co., Inc.; Meso Scale Diagnostics, LLC.; NeuroRx Research; Neurotrack Technologies; Novartis Pharmaceuticals Corporation; Pfizer Inc.; Piramal Imaging; Servier; Takeda Pharmaceutical Company; and Transition Therapeutics. The Canadian Institutes of Health Research is providing funds to support ADNI clinical sites in Canada. Private sector 
contributions are facilitated by the Foundation for the National Institutes of Health (http://www.fnih.org). The grantee organization is the Northern California Institute for Research and Education, and the study is coordinated by the Alzheimer's Therapeutic Research Institute at the University of Southern California. ADNI data are disseminated by the Laboratory for Neuro Imaging at the University of Southern California.

Authors' disclosures available online (http://j-alz. com/manuscript-disclosures/16-1201r2).

\section{SUPPLEMENTARY MATERIAL}

The supplementary material is available in the electronic version of this article: http://dx.doi.org/ 10.3233/JAD-161201.

\section{REFERENCES}

[1] Petersen RC, Smith GE, Waring SC, Ivnik RJ, Tangalos EG, Kokmen E (1999) Mild cognitive impairment: Clinical characterization and outcome. Arch Neurol 56, 303-308.

[2] Manly JJ, Tang M-X, Schupf N, Stern Y, Vonsattel J-PG, Mayeux R (2008) Frequency and course of mild cognitive impairment in a multiethnic community. Ann Neurol 63, 494-506.

[3] Jack CR, Petersen RC, Xu YC, O'Brien PC, Smith GE, Ivnik RJ, Boeve BF, Waring SC, Tangalos EG, Kokmen E (1999) Prediction of AD with MRI-based hippocampal volume in mild cognitive impairment. Neurology 52, 1397-1397.

[4] Risacher SL, Saykin AJ, West JD, Shen L, Firpi HA, McDonald BC (2009) Baseline MRI predictors of conversion from MCI to probable AD in the ADNI cohort. Curr Alzheimer Res 6, 347-361.

[5] Davatzikos C, Xu F, An Y, Fan Y, Resnick SM (2009) Longitudinal progression of Alzheimer's-like patterns of atrophy in normal older adults: The SPARE-AD index. Brain 132, 2026-2035.

[6] Mosconi L, Berti V, Glodzik L, Pupi A, De Santi S, de Leon MJ (2010) Pre-clinical detection of Alzheimer's disease using FDG-PET, with or without amyloid imaging. J Alzheimers Dis 20, 843-854.

[7] Chapman RM, Mapstone M, McCrary JW, Gardner MN, Porsteinsson A, Sandoval TC, Guillily MD, DeGrush E, Reilly LA (2011) Predicting conversion from mild cognitive impairment to Alzheimer's disease using neuropsychological tests and multivariate methods. J Clin Exp Neuropsychol 33, 187-199.

[8] Dickerson BC, Sperling RA, Hyman BT, Albert MS, Blacker D (2007) Clinical prediction of Alzheimer disease dementia across the spectrum of mild cognitive impairment. Arch Gen Psychiatry 64, 1443-1450.

[9] Fleisher AS, Sowell BB, Taylor C, Gamst AC, Petersen RC, Thal LJ, Alzheimer's Disease Cooperative Study (2007) Clinical predictors of progression to Alzheimer disease in amnestic mild cognitive impairment. Neurology 68, 15881595.
[10] Fjell AM, Walhovd KB, Fennema-Notestine C, McEvoy LK, Hagler DJ, Holland D, Brewer JB, Dale AM; Alzheimer's Disease Neuroimaging Initiative (2010) CSF biomarkers in prediction of cerebral and clinical change in mild cognitive impairment and Alzheimer's disease. J Neurosci 30, 2088-2101.

[11] Shaw LM, Vanderstichele H, Knapik-Czajka M, Clark CM, Aisen PS, Petersen RC, Blennow K, Soares H, Simon A, Lewczuk P, Dean R, Siemers E, Potter W, Lee VM-Y, Trojanowski JQ (2009) Cerebrospinal fluid biomarker signature in Alzheimer's disease neuroimaging initiative subjects. Ann Neurol 65, 403-413.

[12] Tabert MH, Manly JJ, Liu X, Pelton GH, Rosenblum S, Jacobs M, Zamora D, Goodkind M, Bell K, Stern Y, Devanand DP (2006) NEuropsychological prediction of conversion to alzheimer disease in patients with mild cognitive impairment. Arch Gen Psychiatry 63, 916-924.

[13] Barnes DE, Cenzer IS, Yaffe K, Ritchie CS, Lee SJ, Alzheimer's Disease Neuroimaging Initiative (2014) A point-based tool to predict conversion from mild cognitive impairment to probable Alzheimer's disease. Alzheimers Dement 10, 646-655.

[14] Lo RY, Hubbard AE, Shaw LM, Trojanowski JQ, Petersen RC, Aisen PS, Weiner MW, Jagust WJ, Alzheimer's Disease Neuroimaging Initiative (2011) Longitudinal change of biomarkers in cognitive decline. Arch Neurol 68, 1257 1266.

[15] Zhang D, Shen D, Alzheimer's Disease Neuroimaging Initiative (2012) Predicting future clinical changes of MCI patients using longitudinal and multimodal biomarkers. PLoS One 7, e33182.

[16] Vellas B, Carrillo MC, Sampaio C, Brashear HR, Siemers E, Hampel H, Schneider LS, Weiner M, Doody R, Khachaturian Z, Cedarbaum J, Grundman M, Broich K, Giacobini E, Dubois B, Sperling R, Wilcock GK, Fox N, Scheltens P, Touchon J, Hendrix S, Andrieu S, Aisen P, EU/US/CTAD Task Force Members (2013) Designing drug trials for Alzheimer's disease: What we have learned from the release of the phase III antibody trials: A report from the EU/US/CTAD Task Force. Alzheimers Dement 9, 438-444.

[17] Bateman RJ, Benzinger TL, Berry S, Clifford DB, Duggan C, Fagan AM, Fanning K, Farlow MR, Hassenstab J, McDade EM, Mills S, Paumier K, Quintana M, Salloway SP, Santacruz A, Schneider LS, Wang G, Xiong C (2017) The DIAN-TU Next Generation Alzheimer's prevention trial: Adaptive design and disease progression model. Alzheimers Dement 13, 8-19.

[18] Lenz RA, Pritchett YL, Berry SM, Llano DA, Han S, Berry DA, Sadowsky CH, Abi-Saab WM, Saltarelli MD (2015) Adaptive, dose-finding phase 2 trial evaluating the safety and efficacy of ABT-089 in mild to moderate Alzheimer disease. Alzheimer Dis Assoc Disord 29, 192-199.

[19] Faucett CL, Thomas DC (1996) Simultaneously modelling censored survival data and repeatedly measured covariates: A Gibbs sampling approach. Stat Med 15, 1663-1685.

[20] Wulfsohn MS, Tsiatis AA (1997) A joint model for survival and longitudinal data measured with error. Biometrics $\mathbf{5 3}$, 330-339.

[21] Landau SM, Harvey D, Madison CM, Koeppe RA, Reiman EM, Foster NL, Weiner MW, Jagust WJ; Alzheimer's Disease Neuroimaging Initiative (2011) Associations between cognitive, functional, and FDG-PET measures of decline in AD and MCI. Neurobiol Aging 32, 1207-1218. 
[22] Jagust WJ, Bandy D, Chen K, Foster NL, Landau SM, Mathis CA, Price JC, Reiman EM, Skovronsky D, Koeppe RA; Alzheimer's Disease Neuroimaging Initiative (2010) The ADNI PET Core. Alzheimers Dement 6, 221-229.

[23] Landau SM, Mintun MA, Joshi AD, Koeppe RA, Petersen RC, Aisen PS, Weiner MW, Jagust WJ; Alzheimer's Disease Neuroimaging Initiative (2012) Amyloid deposition, hypometabolism, and longitudinal cognitive decline. Ann Neurol 72, 578-586.

[24] Fennema-Notestine C, Hagler DJ, McEvoy LK, Fleisher AS, Wu EH, Karow DS, Dale AM (2009) Structural MRI biomarkers for preclinical and mild Alzheimer's disease. Hum Brain Mapp 30, 3238-3253.

[25] Rizopoulos D (2012) Joint Models for Longitudinal and Time-to-Event Data: With Applications in R, CRC Press.

[26] Ibrahim JG, Chu H, Chen LM (2010) Basic concepts and methods for joint models of longitudinal and survival data. J Clin Oncol 28, 2796-2801.

[27] Rizopoulos D (2011) Dynamic Predictions and prospective accuracy in joint models for longitudinal and time-to-event data. Biometrics 67, 819-829.

[28] Stone M (1977) Asymptotics for and against crossvalidation. Biometrika 64, 29-35.

[29] Rizopoulos D (2010) JM: An R package for the joint modelling of longitudinal and time-to-event data. J Stat Softw 35, 1-33.

[30] Fleisher AS, Sun S, Taylor C, Ward CP, Gamst AC, Petersen RC, Jack CR, Aisen PS, Thal LJ (2008) Volumetric MRI vs clinical predictors of Alzheimer disease in mild cognitive impairment. Neurology 70, 191-199.

[31] Schmand B, Huizenga HM, van Gool WA (2010) Metaanalysis of CSF and MRI biomarkers for detecting preclinical Alzheimer's disease. Psychol Med 40, 135-145.

[32] Albert M, Soldan A, Gottesman R, McKhann G, Sacktor N, Farrington L, Grega M, Turner R, Lu Y, Li S, Wang M-C, Selnes O (2014) Cognitive changes preceding clinical symptom onset of mild cognitive impairment and relationship to ApoE genotype. Curr Alzheimer Res 11, 773-784.
[33] Jack CR, Knopman DS, Jagust WJ, Shaw LM, Aisen PS, Weiner MW, Petersen RC, Trojanowski JQ (2010) Hypothetical model of dynamic biomarkers of the Alzheimer's pathological cascade. Lancet Neurol 9, 119.

[34] Li S, Okonkwo O, Albert M, Wang M-C (2013) Variation in variables that predict progression from MCI to AD dementia over duration of follow-up. Am J Alzheimers Dis (Columbia) 2, 12-28.

[35] Cui Y, Liu B, Luo S, Zhen X, Fan M, Liu T, Zhu W, Park M, Jiang T, Jin JS, Alzheimer's Disease Neuroimaging Initiative (2011) Identification of conversion from mild cognitive impairment to Alzheimer's disease using multivariate predictors. PLoS One 6, e21896.

[36] Gomar JJ (2011) Utility of combinations of biomarkers, cognitive markers, and risk factors to predict conversion from mild cognitive impairment to Alzheimer disease in patients in the Alzheimer's Disease Neuroimaging Initiative. Arch Gen Psychiatry 68, 961.

[37] Ewers M, Walsh C, Trojanowski JQ, Shaw LM, Petersen RC, Jack CR, Feldman HH, Bokde ALW, Alexander GE, Scheltens P, Vellas B, Dubois B, Weiner M, Hampel $\mathrm{H}$; North American Alzheimer's Disease Neuroimaging Initiative (ADNI) (2012) Prediction of conversion from mild cognitive impairment to Alzheimer's disease dementia based upon biomarkers and neuropsychological test performance. Neurobiol Aging 33, 1203-1214.e2.

[38] He B, Luo S (2016) Joint modeling of multivariate longitudinal measurements and survival data with applications to Parkinson's disease. Stat Methods Med Res 25, 1346-1358.

[39] Wang J, Luo S, Li L (2016) Dynamic prediction for multiple repeated measures and event time data: An application to Parkinson's disease. arXiv:1603.06476.

[40] Edmonds EC, Delano-Wood L, Galasko DR, Salmon DP, Bondi MW; Alzheimer's Disease Neuroimaging Initiative (2015) Subtle cognitive decline and biomarker staging in preclinical Alzheimer's disease. J Alzheimers Dis 47, 231-242. 